\title{
A Fixed-Point Problem with Mixed-Type Contractive Condition
}

\author{
Calogero Vetro*
}

ABSTRACT. We consider a fixed-point problem for mappings involving a mixed-type contractive condition in the setting of metric spaces. Precisely, we establish the existence and uniqueness of fixed point using the recent notions of $F$-contraction and $(H, \varphi)$-contraction.

Keywords: Fixed point, metric space, mixed-type contractive condition.

2010 Mathematics Subject Classification: 47H10, 54H25.

\section{INTRODUCTION}

Let $(X, d)$ be a complete metric space and let $T: X \rightarrow X$ be a mapping. In this paper, we study the following fixed-point problem:

$$
\left\{\begin{array}{l}
\text { Find } z \in X \text { such that } T z=z, \\
\tau+F(H(d(T x, T y), \varphi(T x), \varphi(T y))) \leq F(H(d(x, y), \varphi(x), \varphi(y))), \quad \tau>0,
\end{array}\right.
$$

for all $x, y \in X$ such that $H(d(T x, T y), \varphi(T x), \varphi(T y))>0$.

This problem is determined by using three functions, namely $F: \mathbb{R}^{+} \rightarrow \mathbb{R}, H:\left[0,+\infty\left[^{3} \rightarrow\right.\right.$ $[0,+\infty[$ and $\varphi: X \rightarrow[0,+\infty[$, with suitable properties (properly stated in Section 2).

Existence results of solutions for different fixed-point problems were proved by many authors. Here, we mention Banach [1] (the pioneering paper on contractions), Wardowski [12] ( $F$-contractions, where $F$ belongs to an appropriate family of functions, namely $\mathcal{F}$ in the sequel), Reem-Reich-Zaslavski [6] (contractive nonself-mappings), Reich-Zaslavski [7] (Matkowski contractions), Reich-Zaslavski [8] (Rakotch contractions), Jleli-Samet-Vetro [2] ( $H, \varphi)$-contractions, where $H$ belongs to an appropriate family of functions, namely $\mathcal{H}$ in the sequel). Also, we recall the comprehensive book of Rus-Petruşel-Petruşel [9], and some results establishing the existence and uniqueness of fixed points that are zeros of a given function (see Samet-VetroVetro [10] and Vetro-Vetro [11]). Finally, we quote the important results of Anthony To-Ming Lau and coworkers, who in a series of remarkable papers discussed the fixed-point property of mappings (see, for example, [3, 4,5] and the references therein).

In this paper, we establish two existence and uniqueness results using a new type of contractive condition working on the classical metric space. In particular, we show that under appropriate assumptions these fixed points are zeros of given functions. Also, we give an example to support the new contractive condition. Precisely, the main result of our paper is the following existence and uniqueness theorem for problem $\left(P_{\tau}\right)$ :

Received: 04.01.2020; Accepted: 12.02.2020; Published Online: 25.02.2020

*Corresponding author: Calogero Vetro; calogero.vetro@unipa.it

DOI: $10.33205 / \mathrm{cma} .684638$ 
Theorem 1.1. If $\varphi: X \rightarrow\left[0,+\infty\left[\right.\right.$ is a lower semicontinuous function, then problem $\left(P_{\tau}\right)$ admits a unique solution $z$ such that $\varphi(z)=0$.

\section{PRELIMINARIES}

Let $(X, d)$ be a metric space and let $T: X \rightarrow X$ be a mapping. We introduce the notation and notions needed in the sequel of this paper. For convenience of the reader, we start with basic facts concerning $F$-contractions and $(H, \varphi)$-contractions.

Definition 2.1. Let $\mathcal{F}$ be the family of all functions $F: \mathbb{R}^{+} \rightarrow \mathbb{R}$ satisfying the following conditions:

$\left(F_{1}\right) F$ is nondecreasing,

$\left(F_{2}\right)$ for every sequence $\left\{\alpha_{n}\right\}$ of positive numbers $\lim _{n \rightarrow+\infty} \alpha_{n}=0$ if and only if $\lim _{n \rightarrow+\infty} F\left(\alpha_{n}\right)=$ $-\infty$,

$\left(F_{3}\right)$ there exists $\left.k \in\right] 0,1\left[\right.$ such that $\lim _{\alpha \rightarrow 0^{+}} \alpha^{k} F(\alpha)=0$.

Now, the mapping $T$ is said to be an $F$-contraction if there exists $\tau>0$ and $F \in \mathcal{F}$ such that

$$
\tau+F(d(T x, T y)) \leq F(d(x, y)) \quad \text { for all } x, y \in X, d(T x, T y) \neq 0 .
$$

From (2.1), since $\tau>0$, we infer that

$$
F(d(T x, T y))<F(d(x, y)) \quad \text { for all } x, y \in X, T x \neq T y .
$$

Using the property $\left(F_{1}\right)$ of the function $F$, we deduce that

$$
d(T x, T y)<d(x, y) \quad \text { for all } x, y \in X, T x \neq T y .
$$

So, each $F$-contraction is a continuous mapping. Using this notion, Wardowski (see [12]) established the following significant result.

Theorem 2.2. Let $(X, d)$ be a complete metric space and let $T: X \rightarrow X$ be an F-contraction. Then $T$ has a unique fixed point $z \in X$ and for every $x_{0} \in X$ the sequence $\left\{T^{n} x_{0}\right\}$ is convergent to $z$.

The functions from $\mathbb{R}^{+}$to $\mathbb{R}$ defined by

(i) $F(t)=\ln t$ for all $t \in \mathbb{R}^{+}$,

(ii) $F(t)=t+\ln t$ for all $t \in \mathbb{R}^{+}$

are classical examples of functions belonging to $\mathcal{F}$.

In [2], Jleli et al. introduced a family $\mathcal{H}$ of functions $H:[0,+\infty]^{3} \rightarrow[0,+\infty[$ satisfying the following conditions:

$\left(H_{1}\right) \max \{a, b\} \leq H(a, b, c)$ for all $a, b, c \in[0,+\infty[$,

$\left(H_{2}\right) H(0,0,0)=0$,

$\left(H_{3}\right) H$ is continuous.

Some examples of functions belonging to $\mathcal{H}$ are given as follows:

(i) $H(a, b, c)=a+b+c$ for all $a, b, c \in[0,+\infty[$,

(ii) $H(a, b, c)=\max \{a, b\}+c$ for all $a, b, c \in[0,+\infty[$,

(iii) $H(a, b, c)=a+b+a b+c$ for all $a, b, c \in[0,+\infty[$.

Using a function $H \in \mathcal{H}$, the authors of [2] introduced the following notion of $(H, \varphi)$ contraction.

Definition 2.2. Let $(X, d)$ be a metric space, $\varphi: X \rightarrow[0,+\infty[$ be a given function and $H \in \mathcal{H}$. Then, $T: X \rightarrow X$ is called $a(H, \varphi)$-contraction with respect to the metric $d$ if and only if

$$
H(d(T x, T y), \varphi(T x), \varphi(T y)) \leq k H(d(x, y), \varphi(x), \varphi(y)) \quad \text { for all } x, y \in X,
$$

for some constant $k \in] 0,1[$. 
Now, we set

$$
\begin{aligned}
& Z_{\varphi}:=\{x \in X: \varphi(x)=0\}, \\
& F_{T}:=\{x \in X: T x=x\} .
\end{aligned}
$$

Furthermore, we say that $T$ is a $\varphi$-Picard operator if and only if the following condition holds:

$$
F_{T} \cap Z_{\varphi}=\{z\} \text { and } T^{n} x \rightarrow z \text {, as } n \rightarrow+\infty \text {, for each } x \in X .
$$

Consequently, we recall the following theorem of [2].

Theorem 2.3. Let $(X, d)$ be a complete metric space, $\varphi: X \rightarrow[0,+\infty[$ be a given function and $H \in \mathcal{H}$. Suppose that the following conditions hold:

$\left(A_{1}\right) \varphi$ is lower semi-continuous,

$\left(A_{2}\right) T: X \rightarrow X$ is a $(H, \varphi)$-contraction with respect to the metric $d$.

Then

(i) $F_{T} \subset Z_{\varphi}$

(ii) $T$ is a $\varphi$-Picard operator,

(iii) for all $x \in X$ and for all $n \in \mathbb{N}$, we have

$$
d\left(T^{n} x, z\right) \leq \frac{k^{n}}{1-k} H(d(T x, x), \varphi(T x), \varphi(x)),
$$

where $\{z\}=F_{T} \cap Z_{\varphi}=F_{T}$.

\section{MAin RESUlts}

Let $X \neq \emptyset, T: X \rightarrow X, x_{0} \in X$ and $x_{n}=T x_{n-1}$ for all $n \in \mathbb{N}$. Then, we call $\left\{x_{n}\right\}$ a sequence of Picard starting at $x_{0}$. In this section, we state and prove our results (Theorems 1.1 and 3.5), using a new mixed-type contraction. Precisely, we establish the existence and uniqueness of fixed point that are zeros of a given function.

Definition 3.3. Let $(X, d)$ be a metric space and let $T: X \rightarrow X$ be a mapping. The mapping $T$ is called an $F$-H-contraction if there exist a function $F \in \mathcal{F}$, a function $H \in \mathcal{H}$, a real number $\tau>0$ and a function $\varphi: X \rightarrow[0,+\infty[$ such that

$$
\tau+F(H(d(T x, T y), \varphi(T x), \varphi(T y))) \leq F(H(d(x, y), \varphi(x), \varphi(y)))
$$

for all $x, y \in X$ with $H(d(T x, T y), \varphi(T x), \varphi(T y))>0$.

We remark that every $F$-contraction is an $F$ - $H$-contraction if we choose $H \in \mathcal{H}$ defined by $H(a, b, c)=a+b+c$ for all $a, b, c \in[0,+\infty[$, and $\varphi: X \rightarrow[0,+\infty$ [ defined by $\varphi(x)=0$ for all $x \in X$. The following is an example of an $F-H$-contraction that is not an $F$-contraction, so that the new definition is a proper extension of the previous one.

Example 3.1. Let $X=[0,1]$ endowed with the usual metric $d(x, y)=|x-y|$ for all $x, y \in X$. Consider the mapping $T: X \rightarrow X$ defined by

$$
T x= \begin{cases}\frac{x}{2} & \text { if } x \in[0,1[, \\ \frac{3}{4} & \text { if } x=1 .\end{cases}
$$

Clearly, $T$ is not an F-contraction since it is not continuous. Now, $T$ is an $F$-H-contraction with respect to the functions $F \in \mathcal{F}$ defined by $F(t)=\ln t$ for all $t>0, H \in \mathcal{H}$ defined by $H(a, b, c)=a+b+c$ for all $a, b, c \in[0,+\infty[$, and $\varphi: X \rightarrow[0,+\infty[$ defined by $\varphi(x)=x$ for all $x \in X$. 
Indeed, for all $x, y \in X$ with $0<x \leq y<1$ or $0=x<y<1$, we have

$$
\begin{aligned}
& F(H(d(T x, T y)+\varphi(T x)+\varphi(T y)))=\ln y, \\
& F(H(d(x, y)+\varphi(x)+\varphi(y)))=\ln 2 y,
\end{aligned}
$$

and for all $x \in[0,1]$ and $y=1$, we have

$$
\begin{aligned}
& F(H(d(T x, T y)+\varphi(T x)+\varphi(T y)))=\ln \frac{3}{2}, \\
& F(H(d(x, x)+\varphi(x)+\varphi(x)))=\ln 2 .
\end{aligned}
$$

Consequently, for every $0<\tau<\ln \frac{4}{3}$, we infer that

$$
\tau+F(H(d(T x, T y)+\varphi(T x)+\varphi(T y))) \leq F(H(d(x, y)+\varphi(x)+\varphi(y)))
$$

for all $x, y \in X$ with $H(d(T x, T y)+\varphi(T x)+\varphi(T y))>0$, that is, $T$ is an $F$-H-contraction.

We establish the following auxiliary lemma.

Lemma 3.1. Let $(X, d)$ be a metric space and let $T: X \rightarrow X$ be an $F$-H-contraction with respect to the functions $F \in \mathcal{F}, H \in \mathcal{H}, \varphi: X \rightarrow\left[0,+\infty\left[\right.\right.$ and the real number $\tau>0$. If $\left\{x_{n}\right\}$ is a sequence of Picard starting at $x_{0} \in X$, then

$$
\lim _{n \rightarrow+\infty} H\left(d\left(x_{n-1}, x_{n}\right), \varphi\left(x_{n-1}\right), \varphi\left(x_{n}\right)\right)=0,
$$

and hence

$$
\lim _{n \rightarrow+\infty} d\left(x_{n-1}, x_{n}\right)=0 \text { and } \lim _{n \rightarrow+\infty} \varphi\left(x_{n}\right)=0 .
$$

Proof. Let $x_{0}$ be an arbitrary point in $X$ and let $\left\{x_{n}\right\}$ be a sequence of Picard starting at $x_{0} \in X$. Firstly, we assume that there exists $k \in \mathbb{N}$ such that $x_{k-1}=x_{k}$, then $x_{n}=x_{k}$ for all $n \geq k$. We claim that $H\left(d\left(x_{k-1}, x_{k}\right), \varphi\left(x_{k-1}\right), \varphi\left(x_{k}\right)\right)=0$.

Assume the contrary, that is, suppose $H\left(d\left(x_{k-1}, x_{k}\right), \varphi\left(x_{k-1}\right), \varphi\left(x_{k}\right)\right)>0$. We remark that

$$
H\left(d\left(x_{k}, x_{k+1}\right), \varphi\left(x_{k}\right), \varphi\left(x_{k+1}\right)\right)=H\left(d\left(x_{k-1}, x_{k}\right), \varphi\left(x_{k-1}\right), \varphi\left(x_{k}\right)\right)>0 .
$$

Using (3.2) with $x=x_{k-1}$ and $y=x_{k}$, we get

$$
\begin{aligned}
\left.\tau+F\left(H\left(d\left(T x_{k-1}, T x_{k}\right)\right), \varphi\left(T x_{k-1}\right), \varphi\left(T x_{k}\right)\right)\right) & =\tau+F\left(H\left(0, \varphi\left(x_{k-1}\right), \varphi\left(x_{k-1}\right)\right)\right) \\
& \leq F\left(H\left(0, \varphi\left(x_{k-1}\right), \varphi\left(x_{k-1}\right)\right)\right),
\end{aligned}
$$

which is a contradiction, since $\tau>0$. So, $H\left(d\left(x_{n-1}, x_{n}\right), \varphi\left(x_{n-1}\right), \varphi\left(x_{n}\right)\right)=0$ for all $n \in \mathbb{N}$ with $n \geq k$. This ensures that (3.3) holds and, by the property $\left(H_{1}\right)$ of the function $H,(3.4)$ holds too.

Then, it is not restrictive to suppose that $x_{n-1} \neq x_{n}$ for all $n \in \mathbb{N}$. By the property $\left(H_{1}\right)$ of the function $H$, we obtain that

$$
H\left(d\left(x_{n-1}, x_{n}\right), \varphi\left(x_{n-1}\right), \varphi\left(x_{n}\right)\right) \geq d\left(x_{n-1}, x_{n}\right)>0 \quad \text { for all } n \in \mathbb{N} .
$$

Using (3.2), with $x=x_{n-1}$ and $y=x_{n}$, we deduce that

$$
\tau+F\left(H\left(d\left(T x_{n-1}, T x_{n}\right), \varphi\left(T x_{n-1}\right), \varphi\left(T x_{n}\right)\right)\right) \leq F\left(H\left(d\left(x_{n-1}, x_{n}\right), \varphi\left(x_{n-1}\right), \varphi\left(x_{n}\right)\right)\right)
$$

for all $n \in \mathbb{N}$. The above inequality shows that

$$
F\left(H\left(d\left(x_{n}, x_{n+1}\right), \varphi\left(x_{n}\right), \varphi\left(x_{n+1}\right)\right)\right)<F\left(H\left(d\left(x_{n-1}, x_{n}\right), \varphi\left(x_{n-1}\right), \varphi\left(x_{n}\right)\right)\right)
$$

for all $n \in \mathbb{N}$. Then, the property $\left(F_{1}\right)$ of the function $F$ implies that the sequence

$$
\left\{H\left(d\left(x_{n-1}, x_{n}\right), \varphi\left(x_{n-1}\right), \varphi\left(x_{n}\right)\right)\right\}
$$


is a decreasing sequence of positive real numbers. So, there exists some $l \geq 0$ such that

$$
\lim _{n \rightarrow+\infty} H\left(d\left(x_{n-1}, x_{n}\right), \varphi\left(x_{n-1}\right), \varphi\left(x_{n}\right)\right)=l .
$$

If $l=0$, then the property $\left(H_{1}\right)$ of the function $H$ gives us

$$
\lim _{n \rightarrow+\infty} d\left(x_{n-1}, x_{n}\right)=0 \text { and } \lim _{n \rightarrow+\infty} \varphi\left(x_{n-1}\right)=0 .
$$

Now, suppose $l>0$. Using (3.2), with $x=x_{n-1}$ and $y=x_{n}$, we get

$$
\begin{aligned}
F\left(H\left(d\left(x_{n}, x_{n+1}\right), \varphi\left(x_{n}\right), \varphi\left(x_{n+1}\right)\right)\right) & \leq F\left(H\left(d\left(x_{n-1}, x_{n}\right), \varphi\left(x_{n-1}\right), \varphi\left(x_{n}\right)\right)\right)-\tau \\
& \leq F\left(H\left(d\left(x_{0}, x_{1}\right), \varphi\left(x_{0}\right), \varphi\left(x_{1}\right)\right)\right)-n \tau
\end{aligned}
$$

for all $n \in \mathbb{N}$. From the previous inequality, passing to the limit as $n \rightarrow+\infty$, we obtain

$$
\lim _{n \rightarrow+\infty} F\left(H\left(d\left(x_{n}, x_{n+1}\right), \varphi\left(x_{n}\right), \varphi\left(x_{n+1}\right)\right)\right)=-\infty
$$

and, using the property $\left(F_{2}\right)$ of the function $F$, we get

$$
\lim _{n \rightarrow+\infty} H\left(d\left(x_{n}, x_{n+1}\right), \varphi\left(x_{n}\right), \varphi\left(x_{n+1}\right)\right)=0,
$$

which leads to contradiction and hence $l=0$. So, (3.3) and (3.4) hold.

Remark 3.1. Note that in the proof of Lemma 3.1, we use only the conditions $\left(F_{1}\right)$ and $\left(F_{2}\right)$.

Now, we are ready to give the proof of Theorem 1.1. For reader convenience, we restate Theorem 1.1 in a classical fixed-point form.

Theorem 3.4. Let $(X, d)$ be a complete metric space and let $T: X \rightarrow X$ be an $F$-H-contraction with respect to the functions $F \in \mathcal{F}, H \in \mathcal{H}$, the real number $\tau>0$ and a lower semicontinuous function $\varphi: X \rightarrow[0,+\infty[$ such that (3.2) holds, that is,

$$
\tau+F(H(d(T x, T y), \varphi(T x), \varphi(T y))) \leq F(H(d(x, y), \varphi(x), \varphi(y)))
$$

for all $x, y \in X$ with $H(d(T x, T y), \varphi(T x), \varphi(T y))>0$. Then, $T$ has a unique fixed point $z$ such that $\varphi(z)=0$.

Proof. We start with the proof of fixed-point uniqueness. Arguing by contradiction, we suppose that there exist $z, w \in X$ such that $z=T z, w=T w$ and $z \neq w$ (that is, $T$ admits two distinct fixed points). The hypothesis $z \neq w$ ensures, by the property $\left(H_{1}\right)$ of the function $H$, that

$$
H(d(T z, T w), \varphi(T z), \varphi(T w)) \geq d(T z, T w)=d(z, w)>0 .
$$

Now, using (3.2), with $x=z$ and $y=w$, we get that

$$
\begin{aligned}
\tau+F(H(d(T z, T w), \varphi(T z), \varphi(T w))) & =\tau+F(H(d(z, w), \varphi(z), \varphi(w))) \\
& \leq F(H(d(z, w), \varphi(z), \varphi(w))) .
\end{aligned}
$$

Clearly, this is a contradiction, and hence we have, $w=z$. So, we obtain the claim.

The next step is to establish the existence of a fixed point. We consider a point $x_{0} \in X$. Let $\left\{x_{n}\right\}$ be a sequence of Picard starting at $x_{0}$. We stress that if $x_{k-1}=x_{k}$ for some $k \in \mathbb{N}$, then $z=x_{k-1}=x_{k}=T x_{k-1}=T z$, that is, $z$ is a fixed point of $T$ such that $\varphi(z)=0$. In fact, by Lemma 3.1, $H\left(d\left(x_{k-1}, x_{k}\right), \varphi\left(x_{k-1}\right), \varphi\left(x_{k}\right)\right)=0$ and by the property $\left(H_{1}\right)$ of the function $H$, we have $\varphi(z)=0$. So, we can suppose that $x_{n-1} \neq x_{n}$ for every $n \in \mathbb{N}$.

Now, we prove that $\left\{x_{n}\right\}$ is a Cauchy sequence. By Lemma 3.1, we say that

$$
0<h_{n-1}=H\left(d\left(x_{n-1}, x_{n}\right), \varphi\left(x_{n-1}\right), \varphi\left(x_{n}\right)\right) \rightarrow 0 \quad \text { as } n \rightarrow+\infty \text {. }
$$


The property $\left(F_{3}\right)$ of the function $F$ ensures that there exists $\left.k \in\right] 0,1\left[\right.$ such that $h_{n}^{k} F\left(h_{n}\right) \rightarrow 0$ as $n \rightarrow+\infty$. Using (3.2), with $x=x_{n-1}$ and $y=x_{n}$, we get

$$
\begin{aligned}
F\left(H\left(d\left(x_{n}, x_{n+1}\right), \varphi\left(x_{n}\right), \varphi\left(x_{n+1}\right)\right)\right) & \leq F\left(H\left(d\left(x_{n-1}, x_{n}\right), \varphi\left(x_{n-1}\right), \varphi\left(x_{n}\right)\right)\right)-\tau \\
& \leq F\left(H\left(d\left(x_{0}, x_{1}\right), \varphi\left(x_{0}\right), \varphi\left(x_{1}\right)\right)\right)-n \tau
\end{aligned}
$$

for all $n \in \mathbb{N}$, that is,

$$
F\left(h_{n}\right) \leq F\left(h_{n-1}\right)-\tau \leq \cdots \leq F\left(h_{0}\right)-n \tau \quad \text { for all } n \in \mathbb{N} .
$$

From

$$
0=\lim _{n \rightarrow+\infty} h_{n}^{k} F\left(h_{n}\right) \leq \lim _{n \rightarrow+\infty} h_{n}^{k}\left(F\left(h_{0}\right)-n \tau\right) \leq 0
$$

we deduce that

$$
\lim _{n \rightarrow+\infty} h_{n}^{k} n=0 .
$$

This ensures that the series $\sum_{n=1}^{+\infty} h_{n}$ is convergent. By the property $\left(H_{1}\right)$ of the function $H$ also the series $\sum_{n=1}^{+\infty} d\left(x_{n}, x_{n+1}\right)$ is convergent, and hence $\left\{x_{n}\right\}$ is a Cauchy sequence. Now, since $(X, d)$ is complete, there exists some $z \in X$ such that

$$
\lim _{n \rightarrow+\infty} x_{n}=z \text {. }
$$

By (3.4), taking into account that $\varphi$ is a lower semicontinuous function, we get

$$
0 \leq \varphi(z) \leq \liminf _{n \rightarrow+\infty} \varphi\left(x_{n}\right)=0,
$$

that is, $\varphi(z)=0$. We assert that $z$ is a fixed point of $T$. Clearly, $z$ is a fixed point of $T$ if there exists a subsequence $\left\{x_{n_{k}}\right\}$ of $\left\{x_{n}\right\}$ such that $x_{n_{k}}=z$ or $T x_{n_{k}}=T z$, for all $k \in \mathbb{N}$. Otherwise, we can assume that $x_{n} \neq z$ and $T x_{n} \neq T z$ for all $n \in \mathbb{N}$. So, using (3.2) with $x=x_{n}$ and $y=z$, we deduce that

$$
\tau+F\left(H\left(d\left(T x_{n}, T z\right), \varphi\left(T x_{n}\right), \varphi(T z)\right)\right) \leq F\left(H\left(d\left(x_{n}, z\right), \varphi\left(x_{n}\right), \varphi(z)\right)\right) .
$$

Since $\tau>0$, this inequality leads to

$$
H\left(d\left(T x_{n}, T z\right), \varphi\left(T x_{n}\right), \varphi(T z)\right)<H\left(d\left(x_{n}, z\right), \varphi\left(x_{n}\right), \varphi(z)\right) \quad \text { for all } n \in \mathbb{N},
$$

and so

$$
\begin{aligned}
d(z, T z) & \leq d\left(z, x_{n+1}\right)+d\left(T x_{n}, T z\right) \\
& \leq d\left(z, x_{n+1}\right)+H\left(d\left(T x_{n}, T z\right), \varphi\left(T x_{n}\right), \varphi(T z)\right) \\
& <d\left(z, x_{n+1}\right)+H\left(d\left(x_{n}, z\right), \varphi\left(x_{n}\right), \varphi(z)\right)
\end{aligned}
$$

for all $n \in \mathbb{N}$.

Finally, letting $n \rightarrow+\infty$ in the above calculations and taking into account that $H$ is continuous in $(0,0,0)$, we deduce that $d(z, T z) \leq H(0,0,0)=0$, that is, $z=T z$.

Imposing that $F$ is a continuous function and relaxing the hypothesis $\left(F_{3}\right)$, we establish the following result.

Theorem 3.5. Let $(X, d)$ be a complete metric space and let $T: X \rightarrow X$ be a mapping. Assume that there exists a continuous function $F$ that satisfies the conditions $\left(F_{1}\right)$ and $\left(F_{2}\right)$, a function $H \in \mathcal{H}, a$ real number $\tau>0$ and a lower semicontinuous function $\varphi: X \rightarrow[0,+\infty[$ such that (3.2) holds, that is,

$$
\tau+F(H(d(T x, T y), \varphi(T x), \varphi(T y))) \leq F(H(d(x, y), \varphi(x), \varphi(y)))
$$

for all $x, y \in X$ with $H(d(T x, T y), \varphi(T x), \varphi(T y))>0$. Then $T$, has a unique fixed point $z$ such that $\varphi(z)=0$. 
Proof. Following the similar arguments as in the proof of Theorem 3.4, we obtain easily the uniqueness of the fixed point. In order to establish the existence of a fixed point, we consider a point $x_{0} \in X$. Let $\left\{x_{n}\right\}$ be a sequence of Picard starting at $x_{0}$. Clearly if $x_{k-1}=x_{k}$ for some $k \in \mathbb{N}$, then $z=x_{k-1}=x_{k}=T x_{k-1}=T z$, that is, $z$ is a fixed point of $T$ such that $\varphi(z)=0$ (see the proof of Theorem 3.4), and so, we have already done.

So, we can suppose that $x_{n-1} \neq x_{n}$ for every $n \in \mathbb{N}$. We claim that $\left\{x_{n}\right\}$ is a Cauchy sequence. We assume for way of contradiction that $\left\{x_{n}\right\}$ is not a Cauchy sequence. Then, there exist a positive real number $\varepsilon$ and two sequences $\left\{m_{k}\right\}$ and $\left\{n_{k}\right\}$ such that

$$
n_{k}>m_{k} \geq k \text { and } d\left(x_{m_{k}}, x_{n_{k}}\right) \geq \varepsilon>d\left(x_{m_{k}}, x_{n_{k}-1}\right) \text { for all } k \in \mathbb{N} \text {. }
$$

By Lemma 3.1 and Remark 3.1, we say that $d\left(x_{n-1}, x_{n}\right) \rightarrow 0, \varphi\left(x_{n}\right) \rightarrow 0$ as $n \rightarrow+\infty$. This implies

$$
\lim _{k \rightarrow+\infty} d\left(x_{m_{k}}, x_{n_{k}}\right)=\lim _{k \rightarrow+\infty} d\left(x_{m_{k}-1}, x_{n_{k}-1}\right)=\varepsilon .
$$

Now, the hypothesis that $d\left(x_{m_{k}}, x_{n_{k}}\right)>\varepsilon$ ensures that

$$
H\left(d\left(x_{m_{k}}, x_{n_{k}}\right), \varphi\left(x_{m_{k}}\right), \varphi\left(x_{n_{k}}\right)\right)>0 \quad \text { for all } k \in \mathbb{N} .
$$

So, taking into account that $H$ is a continuous function, we have

$$
\begin{aligned}
& \lim _{k \rightarrow+\infty} H\left(d\left(x_{m_{k}-1}, x_{n_{k}-1}\right), \varphi\left(x_{m_{k}-1}\right), \varphi\left(x_{n_{k}-1}\right)\right) \\
& =\lim _{k \rightarrow+\infty} H\left(d\left(x_{m_{k}}, x_{n_{k}}\right), \varphi\left(x_{m_{k}}\right), \varphi\left(x_{n_{k}}\right)\right) \\
& =H(\varepsilon, 0,0)>0 .
\end{aligned}
$$

Using again (3.2), with $x=x_{m_{k}-1}$ and $y=x_{n_{k}-1}$, we deduce that

$$
\begin{aligned}
& \tau+F\left(H\left(d\left(x_{m_{k}}, x_{n_{k}}\right), \varphi\left(x_{m_{k}}\right), \varphi\left(x_{n_{k}}\right)\right)\right) \\
& \leq F\left(H\left(d\left(x_{m_{k}-1}, x_{n_{k}-1}\right), \varphi\left(x_{m_{k}-1}\right), \varphi\left(x_{n_{k}-1}\right)\right)\right)
\end{aligned}
$$

for all $k \in \mathbb{N}$. Letting $k \rightarrow+\infty$ in the previous inequality, since the function $F$ is continuous, we get

$$
\tau+F(H(\varepsilon, 0,0))) \leq F(H(\varepsilon, 0,0))),
$$

which leads to contradiction. It follows that $\left\{x_{n}\right\}$ is a Cauchy sequence.

Now, since $(X, d)$ is complete, there exists some $z \in X$ such that

$$
\lim _{n \rightarrow+\infty} x_{n}=z \text {. }
$$

By (3.4), taking into account that $\varphi$ is a lower semicontinuous function, we get

$$
0 \leq \varphi(z) \leq \liminf _{n \rightarrow+\infty} \varphi\left(x_{n}\right)=0,
$$

that is, $\varphi(z)=0$. We assert that $z$ is a fixed point of $T$. Clearly, $z$ is a fixed point of $T$ if there exists a subsequence $\left\{x_{n_{k}}\right\}$ of $\left\{x_{n}\right\}$ such that $x_{n_{k}}=z$ or $T x_{n_{k}}=T z$, for all $k \in \mathbb{N}$. Otherwise, we can assume that $x_{n} \neq z$ and $T x_{n} \neq T z$ for all $n \in \mathbb{N}$. Then, the property $\left(H_{1}\right)$ of the function $H$ ensures that $H\left(d\left(T x_{n}, T z\right), \varphi\left(T x_{n}\right), \varphi(T z)\right)>0$ for all $n \in \mathbb{N}$. So, using (3.2), with $x=x_{n}$ and $y=z$, we deduce that

$$
\tau+F\left(H\left(d\left(T x_{n}, T z\right), \varphi\left(T x_{n}\right), \varphi(T z)\right)\right) \leq F\left(H\left(d\left(x_{n}, z\right), \varphi\left(x_{n}\right), \varphi(z)\right)\right) \text { for all } n \in \mathbb{N} .
$$

Since $\tau>0$, we conclude that

$$
H\left(d\left(T x_{n}, T z\right), \varphi\left(T x_{n}\right), \varphi(T z)\right)<H\left(d\left(x_{n}, z\right), \varphi\left(x_{n}\right), \varphi(z)\right) \quad \text { for all } n \in \mathbb{N},
$$


and so

$$
\begin{aligned}
d(z, T z) & \leq d\left(z, x_{n+1}\right)+d\left(T x_{n}, T z\right) \\
& \leq d\left(z, x_{n+1}\right)+H\left(d\left(T x_{n}, T z\right), \varphi\left(T x_{n}\right), \varphi(T z)\right) \\
& <d\left(z, x_{n+1}\right)+H\left(d\left(x_{n}, z\right), \varphi\left(x_{n}\right), \varphi(z)\right)
\end{aligned}
$$

for all $n \in \mathbb{N}$. Finally, letting $n \rightarrow+\infty$ and taking into account that $H$ is continuous in $(0,0,0)$, we deduce that $d(z, T z) \leq H(0,0,0)=0$, that is, $z=T z$.

\section{REFERENCES}

[1] S. Banach: Sur les opérations dans les ensembles abstraits et leur application aux équations intégrales. Fund. Math. 3 (1922), 133-181.

[2] M. Jleli, B. Samet and C. Vetro: Fixed point theory in partial metric spaces via $\varphi$-fixed point's concept in metric spaces, J. Inequal. Appl. 2014:426 (2014), 9 pp.

[3] A. T.-M. Lau, W. Takahashi: Invariant means and fixed point properties for nonexpansive representations of topological semigroups. Topol. Methods Nonlinear Anal. 5 (1995), 39-57.

[4] A. T.-M. Lau, Y. Zhang: Fixed point properties of semigroups of non-expansive mappings. J. Funct. Anal. 254 (2008), 2534-2554.

[5] A. T.-M. Lau, Y. Zhang: Fixed point properties for semigroups of nonlinear mappings and amenability. J. Funct. Anal. 263 (2012), 2949-2977.

[6] D. Reem, S. Reich and A. J. Zaslavski: Two Results in Metric Fixed Point Theory. J. Fixed Point Theory Appl. 1 (2007), 149-157.

[7] S. Reich, A. J. Zaslavski: A Fixed Point Theorem for Matkowski Contractions. Fixed Point Theory 8 (2007), 303-307.

[8] S. Reich, A. J. Zaslavski: A Note on Rakotch contraction. Fixed Point Theory 9 (2008), 267-273.

[9] I. A. Rus, A. Petruşel and G. Petruşel: Fixed Point Theory. Cluj University Press, Cluj-Napoca (2008).

[10] B. Samet, C. Vetro and F. Vetro: From metric spaces to partial metric spaces. Fixed Point Theory Appl. 2013:5 (2013), $11 \mathrm{pp}$.

[11] C. Vetro, F. Vetro: Metric or partial metric spaces endowed with a finite number of graphs: a tool to obtain fixed point results. Topology Appl. 164 (2014), 125-137.

[12] D. Wardowski: Fixed points of a new type of contractive mappings in complete metric spaces. Fixed Point Theory Appl., 2012:94 (2012), 6 pp.

UNIVERSITY OF PALERMO

DePARTMENT OF MATHEMATiCS AND COMPUTER SCIENCE

VIA ARCHIRAFI 34, 90123, PALERMO, ITALY

ORCID: 0000-0001-5836-6847

E-mail address: calogero.vetro@unipa. it 